\title{
Applications of Soft Chemical Ionisation Mass Spectrometric Instrumentation to Investigate the Human Volatilome for use in the Medical Sciences
}

\author{
V. Ruzsanyi, P. Mochalski, H. Wiesenhofer, K. Unterkofler, C. Ager, and C. A. Mayhew \\ Institute for Breath Research, Leopold-Franzens-Universität Innsbruck, Rathausplatz 4, \\ 6850, Dornbirn, Austria \\ Christopher.Mayhew@uibk.ac.at
}

\begin{abstract}
Soft chemical ionization mass spectrometric techniques, which include proton transfer reaction mass spectrometry and ion mobility spectrometry, employ ions as sensitive analytical probes for use in the identification, detection and monitoring of trace compounds in complex chemical surroundings. These techniques have opened up new and exciting possibilities for applied areas of research with applications to health, food science, the environment and security. Among these is the potential to use the trace volatiles in human breath, emitted from the skin and present in bodily fluids for diagnosing and monitoring diseases, monitoring treatments and examining health in general. This is possible because these volatiles provide a non-invasive window to physiological and metabolic processes occurring within the body. The research being undertaken at the Institute for Breath Research in Dornbirn, Austria is addressing the many current challenges of applying soft chemical ionization mass spectrometry for the analysis of the human volatilome for the benefit of clinical non-invasive diagnostic procedures; including sampling protocols and importantly the assignment of compounds with a high specificity. The unambiguous identification of volatile compounds is needed to provide the necessary information for the development of sensors for the detection of specific compounds for a given clinical application. Although the main focus of this paper will be on our breath analysis research in the health sciences, details of related projects in homeland security and search and rescue operations, namely those involved in discovering humans trapped in buildings or hiding in containers through the volatiles that people emit, are presented.
\end{abstract}

Key words: Breath Analysis, Breath Sampling, VOCs, PTR-MS, IMS

\section{The Human Volatilome}

Hundreds of volatile organic compounds (VOCs) are present in our breath, emitted through our skin and contained within bodily fluids and feces. Collectively, these can be referred to as the human volatilome [1]. This volatilome contains not only volatile metabolites derived endogenously but also those originating from exogenous sources, which independently or together can provide unique non-invasive information on processes occurring within a human body for use in the health sciences [1-3]. These same volatiles can also be used to detect the presence of entrapped or hidden people $[4,5]$. Of course this requires real time sampling and analysis, which can be done quickly and cheaply by sensors. However, before adopting such technology, the volatile compounds need to be unambiguously identified using more powerful analytical techniques. The same is true in the use of VOCs for medical applications, using real time measurements or not. This unambiguous identification is an objective of the research being undertaken at the Institute for Breath Research in Dornbirn, Austria, using not only soft chemical ionization mass spectrometric techniques, such as proton transfer reaction mass spectrometry (PTRMS) and ion mobility spectrometry (IMS), but also standard Gas Chromatography Mass Spectrometry (GC-MS). Once the volatiles, or their patterns, have been determined, machine-learning and pattern recognition algorithms can then be used in sensors to identify disease states. We illustrate here some of our research activities by highlighting results obtained using soft chemical ionization techniques to identify volatiles in the human volatilome.

\section{The Human Volatilome and Medical Uses}

To date the human volatilome research has focused on volatiles contained in breath [1, 2], although some work has also been done on emissions from skin [1, 2] and volatiles in the head space of blood [1, 2], urine [1, 2], fluids in the gut [6], saliva [1] and fecal matter [1,7]. Volatile biomarkers of disease and drugs present in the bloodstream pass from the blood capillaries into the alveoli in the lungs and then are carried out in the breath. The exhaled volatiles on breath provide a non-invasive window into the physiological and metabolic processes occurring within the whole body and to any drugs present in the bloodstream. Thus the development of breath tests for the non-invasive detection of early stage disease and for the monitoring of a disease during treatment has the potential of having significant impact on society in terms of the quality of life and for reducing the cost of health care. Numerous studies have looked for endogenous volatiles that can be related to a specific disease. But this is a challenging task owing to issues of breath sampling and confounding 
factors, such as medication, treatment, diet, age, and environmental factors. There are also issues of applying appropriate statistical analysis when dealing with multiple compound analysis using a small sample set, so that care must be taken to ensure that "voodoo" (coincidental) correlations do not occur. Hence whilst individual studies claim to have provided evidence for the use of endogenous breath volatiles to diagnose diseases few have shown reproducibility and predictability. Exogenous volatiles are easier to use in breath analysis, because of the reduction in confounding and voodoo factors. Their metabolites, or the lack of production of metabolites, give a direct insight into the state of the human body. A recent example is the reduction in the metabolism of limonene owing to chronic liver disease and hence higher concentrations of limonene in breath are found compared to healthy controls [8].

\section{The Human Volatilome for Detecting Entrapped or Hidden People}

A volatile chemical fingerprint can be used to identify the presence of hidden or entrapped people. These volatile species forming the human scent during entrapment can stem from compounds present in human breath or emitted from urine, feces, blood, sweat and skin. Breath volatiles are particularly important because they can obviously be used to differentiate between living and dead victims. Two analytical techniques arguably have the greatest potential in this context of finding humans, namely IMS and electronic sensors. Recently we have used an ion mobility spectrometer coupled with gas chromatography (GC-IMS) to monitor the volatiles released from the human body under conditions that mimic entrapment [5]. Figure 1 provides an illustrative chromatogram from this instrument.

\section{References}

[1] A. Amann, Bde L Costello, W. Miekisch, J. Schubert, B. Buszewski, J. Pleil, N. Ratcliffe, and T. A. Risby, The human volatilome: volatile organic compounds (VOCs) in exhaled breath, skin emanations, urine, feces and saliva, Journal of Breath Research 8(3), 034001 (2014). doi: 10.1088/1752-7155/8/3/034001

[2] A. M. Ellis and C. A. Mayhew, Proton Transfer Reaction Mass Spectrometry Principles and Applications. (2014) John Wiley \& Sons Ltd., UK. doi: $10.1002 / 9781118682883$

[3] S. Sethi, R. Nanda and T. Chakraborty, Clinical Application of Volatile Organic Compound Analysis for Detecting Infectious Diseases, Clinical Microbiology Reviews 26(3), 462-475 (2013). doi: 10.1128/CMR.00020-13

[4] P. Mochalski, V. Ruzsanyi, H. Wiesenhofer, and C. A. Mayhew, Instrumental sensing of trace volatiles - a new promising tool for detecting the presence of entrapped or hidden people, Journal of Breath Research 12, 027107 (2018). doi: 10.1088/1752-7163/aa9769

[5] P. Mochalski, H. Wiesenhofer, M. Allers, S. Zimmermann, A. T. Güntner, N. J. Pineauc, W.

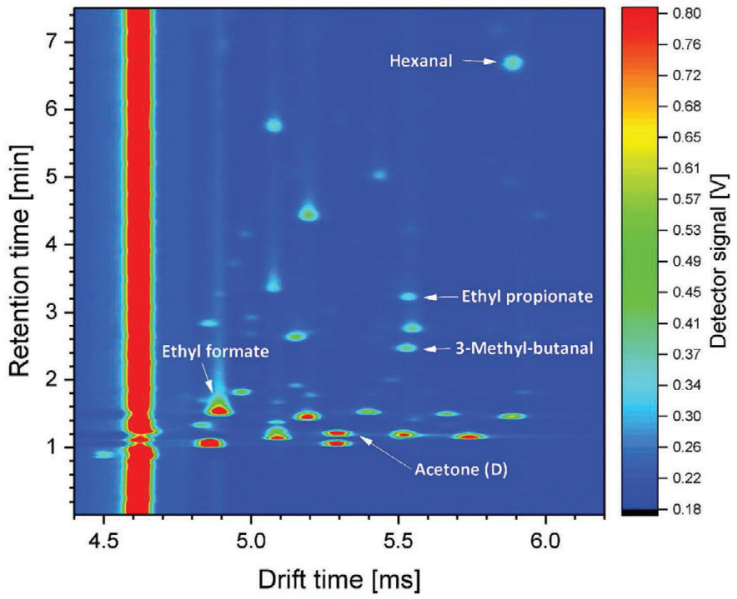

Fig. 1. Fragment of an exemplary 2D GC-IMS chromatogram from the analysis of humanborne VOCs detected in a closed plethysmography chamber containing a human volunteer. Some of the key volatiles are identified.

In total seventeen VOCs were identified and quantified from thirty-five ion mobility peaks corresponding to a human being present. These VOCs are 7 aldehydes (acrolein, 2-methylpropanal, 3-methylbutanal, 2-ethacrolein, n-hexanal, nheptanal, benzaldehyde), 3 ketones (acetone, 2-pentanone, 4methyl-2-pentanone), 5 esters (ethyl formate, ethyl propionate, vinyl butyrate, butyl acetate, ethyl isovalerate), one alcohol (2-methyl-1-propanol) and one organic acid (acetic acid). This proof-of-principle study demonstrates that GC-IMS can be used as a portable field detector of hidden or entrapped people.

Lederer, A. Agapiou, C. A. Mayhew, V. Ruzsanyi, Monitoring of selected skin- and breath-borne volatile organic compounds emitted from the human body using gas chromatography ion mobility spectrometry (GC-IMS), Journal of Chromatography B 1076, 29-34 (2018). doi: 10.1016/j.jchromb.2018.01.013.

[6] M. Lechner, H. P. Colvin, C. Ginzel, C. et al. Headspace screening of fluid obtained from the gut during colonoscopy and breath analysis by proton transfer reaction-mass spectrometry: a novel approach in the diagnosis of gastrointestinal diseases, International Journal of Mass Spectrometry 243, 151-154 (2005). doi: 10.1016/j.jms.2005.02.002

[7] C. A. Batty, M. Cauchi, C. Lourenço, J. O. Hunter, C. Turner, Use of the Analysis of the Volatile Faecal Metabolome in Screening for Colorectal Cancer, PLoS ONE 10(6), e0130301 (2015). doi: 10.1371/journal.pone.0130301

[8] R. Fernández del Río, M. E. O'Hara, A. Holt, P. Pemberton, T. Shah, T. Whitehouse, and C. A. Mayhew, Volatile biomarkers in breath associated with liver cirrhosis - comparisons of pre- and post-liver transplant breath samples, EBioMedicine 2, 1243-1250 (2015). doi: 10.1016/j.ebiom.2015.07.027 